\title{
A COMPUTING METHOD FOR BIFURCATION BOUGHS OF NONLINEAR EIGENVALUE PROBLEMS
}

\author{
BY RENÉ LOZI
}

Communicated by François Treves, July 19, 1975

Bifurcation branches of solutions of nonlinear (differential) equations dependent on a real-parameter is now a well developed theory. (See [4] for general aspects, [3] for existence and [1] for examples.) However, what is known is mostly bifurcate solutions in a neighbourhood of the bifurcation points, and there exist few numerical methods that allow us to obtain these branches.

The method we propose allows us to obtain the "regular" parts of these branches, that means without "critical" points, as solutions of a Cauchy problem in which the real bifurcation parameter is the variable.

1. Definitions. Let $X$ and $Z$ be two real-Banach spaces and $f$ a continuously Fréchet-differentiable mapping from $X \times \mathbf{R}$ to $Z$. $D_{1} f$ denotes the partial derivative in $x$. We seek solutions $(x, y)$ in $X \times \mathbf{R}$ of the following equation:

$$
f(x, y)=0
$$

$y$ is often called the bifurcation parameter. Let $S$ be the set of these solutions and $C$ the subset of $S$ for which $D_{1} f(x, y)$ is not a homeomorphism ( $C$ is the set of "critical solutions"); then every maximal (with respect to the relation of inclusion) connected subset $B$ of $S-C$ is called bifurcation bough.

2. One property of bifurcation boughs. We shall use the following classical result (Lemma 1) to prove the main theorem.

LEмма 1. Let $E$ be a separate, connected, topological space, and $h$ a local homeomorphism from $E$ to $\mathbf{R}$; then $h$ is a homeomorphism from $E$ onto $h(E)$.

The theorem which gives the main property of bifurcation boughs is:

THEOREM 1. For every bifurcation bough B, let I (resp. K) be its projection on $\mathbf{R}$ (resp. X). There exists a unique continuously differentiable mapping $\Psi$ from $I$ to $K$ such that:

(i) The graph of $\Psi$ in $X \times \mathbf{R}$ is $B$.

(ii) $\Psi^{\prime}(y)=-\left[D_{1} f(\Psi(y), y)\right]^{-1} \circ D_{2} f(\Psi(y), y)$.

Proof. At every point $(x, y)$ of $B$, we can apply the implicit function theorem; there exists an open interval $I_{y} \subset \mathrm{R}$ including $y$, and a unique mapping $\varphi$

AMS (MOS) subject classifications (1970). Primary 35A15, 35P15, 65 L05. 
from $I_{y}$ to $X$, such that $\varphi(y)=x$ and $f(\varphi(z), z)=0 \forall z \in I_{y}$; furthermore $\varphi$ is continuously differentiable in $I_{y}$ and its derivative at every point $z$ of $I_{y}$ is given by

$$
\varphi^{\prime}(z)=-\left[D_{1} f(\varphi(z), z)\right]^{-1} \circ D_{2} f(\varphi(z), z) .
$$

Consider now the projection from $X \times \mathbf{R}$ to $\mathbf{R}$ and its restriction $p$ to $B$. Then for every point $(x, y)$ of $B$, there exists an open interval $I_{y} \subset \mathbf{R}$ containing $y$, such that $p$ is a one-to-one mapping from $p^{-1}\left(I_{y}\right)$ onto $I_{y}$. Furthermore in $I_{y}$, there exists an open subinterval $J_{y}$ containing $y$ such that $p^{-1}\left(J_{y}\right) \subset B\left(D_{1} f\right.$ is locally invertible). As $p$ is continuous we are in the situation of Lemma 1. Hence, $p$ is a homeomorphism from B onto $I$. Now, if $p^{\prime}$ denotes the projection from $B$ to $K$ we can conclude defining $\Psi$ by $\Psi(y)=p^{\prime} \circ p^{-1}(y)$.

COROLlaRY 1. Assume the mapping $f$ is $k$-times continuously differentiable in $X \times \mathbf{R}$, then the mapping $\Psi$ of Theorem 1 is $k$-times continuously differentiable.

3. A computing method for bifurcation boughs. Seeing Theorem 1 and Corollary 1, and since the set: $\left\{D_{1} f(x, y) \mid(x, y) \in X \times \mathbf{R}\right.$ with $D_{1} f(x, y)$ homeomorphism $\}$ is open in $L(X, \mathbf{R} ; Z)$, there is an open neighbourhood $V$ in $X \times \mathbf{R}$ of every bifurcation bough where the mapping

$$
(x, y) \mapsto-\left[D_{1} f(x, y)\right]^{-1} \circ D_{2} f(x, y)
$$

is defined and smooth enough. In order to obtain the bifurcation bough containing the point $\left(x_{0}, y_{0}\right)$ we can integrate the differential equation

$$
\left\{\begin{array}{l}
\Psi^{\prime}(y)=-\left[D_{1} f(\Psi(y), y)\right]^{-1} \circ D_{2} f(\Psi(y), y), \\
\Psi\left(y_{0}\right)=x_{0}
\end{array}\right.
$$

using a Runge-Kutta type method (see [2]).

4. Numerical results. We study the equation

$$
s=y t+1 / 4 s^{2}, \quad t=y s+1 / 4 t^{2} ;
$$

here $X=Z=\mathrm{R}^{2}$ and $x=(s, t)$.

The bifurcation points are $(0,0,-1)$ and $(0,0,1)$ in $X \times \mathbf{R}$. The solutions of (3) are

$$
\begin{aligned}
& \text { the trivial solution } s=t=0 \quad \forall y \in \mathbf{R}, \\
& s=t=4(1-y) \quad \forall y \in \mathbf{R}, \\
& \left\{\begin{array}{l}
\text { if } \alpha=\sqrt{-3 y^{2}-2 y+1} \\
s=2(y+1 \pm \alpha) \\
t=2(y+1 \mp \alpha)
\end{array} \quad \forall y \in[-1,1 / 3]\right.
\end{aligned}
$$


We computed these solutions, integrating (2) by a fourth order Runge-Kutta method. The results are in Table 1. " $\delta y$ " denotes the step of the Runge-Kutta method and "Error" the value of $\left|s-s_{r}\right|+\left|t-t_{r}\right|$ where $(s, t)$ are the components of the exact solution, and $\left(s_{r}, t_{r}\right)$ the components of the computed solution for the value $y+\delta y$ of the bifurcation parameter.

TABLE 1

\begin{tabular}{rrrc}
\hline$y_{0}$ & $\delta y$ & $y+\delta y$ & Error \\
\hline 0.00 & -0.10 & -0.10 & $0.82 \times 10^{-5}$ \\
-0.50 & -0.10 & -0.60 & $0.35 \times 10^{-5}$ \\
\hline 0.20 & -0.01 & 0.19 & $0.59 \times 10^{-7}$ \\
0.20 & -0.10 & 0.10 & $0.20 \times 10^{-3}$ \\
0.20 & -0.20 & 0.00 & $0.30 \times 10^{-2}$ \\
0.20 & -0.40 & -0.20 & $0.31 \times 10^{-1}$ \\
\hline
\end{tabular}

We tried this method with many other examples and all the results are equally valid.

\section{BIBLIOGRAPHY}

1. G. Iooss and G. Tronel, Exemples de bifurcation, C. R. Acad. Sci. Paris Sér. A-B 276 (1973), A1353-A1356. MR 47 \#7535.

2. H. B. Keller, Numerical methods for two-point boundary value problems, Blaisdell, Waltham, Mass., 1968. MR 37 \#6038.

3. P. H. Rabinowitz, Some global results for nonlinear eigenvalue problems, J. Functional Analysis 7 (1971), 487-513. MR 46 \#745.

4. D. H. Sattinger, Topics in stability and bifurcation theory, Lecture Notes in Math., vol. 309, Springer-Verlag, Berlin and New York, 1973.

INSTITUT DE MATHEMATIQUES ET SCIENCES PHYSIQUES PARC VALROSE 06434 NICE CEDEX, FRANCE 